\title{
Paraurethral leiomyoma in a woman: a case report
}

\section{Sendhil Coumary A*, Seetesh Ghose, Rupal Samal}

Department of Obstetrics \& Gynecology, Mahatma Gandhi Medical College and Research Institute, Pilliarkuppam, Pondicherry-607402, India

Received: 29 December 2012

Accepted: 6 January 2013

*Correspondence:

Dr. Sendhil Coumary A

E-mail: sendhilcoumary1975@yahoo.in

\section{ABSTRACT}

Paraurethral leiomyomas are rare, benign smooth muscle tumours of the urogenital tract. Most para-urethral tumours occur in the reproductive age group. Its etiology is uncertain and its presentation is variable. Here we present a 45 year old multiparous lady, who approached the out-patient department, with complaints of profuse foul-smelling discharge and a mass per vaginum for 10 days.

Keywords: Paraurethral, Leiomyoma, Enucleation

\section{INTRODUCTION}

Leiomyoma is a benign neoplasm commonly encountered in gynaecological practice. They are derived from smooth muscle cell rests. They are broadly classified as uterine and extra-uterine. A small percentage of Leiomyomas arise from the uterine ligaments, vulva, vagina and paraurethral region. Para-urethral leiomyomas are rare. Literature has reported only few cases till date.

\section{CASE REPORT}

A forty five year old multiparous lady approached the outpatient department, with complaints of profuse foulsmelling discharge and a mass per vaginum for ten days. Her menstrual cycles were regular with normal flow though with dysmenorrhoea. She is married for the past thirty years. She also gives history of dyspareunia over the last six months.

Her general physical examination was unremarkable. An examination of the abdomen and the external genitalia was normal. Speculum examination revealed a fleshy, firm mass, measuring four $\mathrm{x}$ four centimeters, cephalad of the external urethral meatus. It was sessile and non-tender on palpation. On performing valsalva manoeuvre, the mass could be seen outside the introitus. Rest of the vagina and cervix were healthy. Bimanual and per rectal examination was unremarkable. A provisional diagnosis of paraurethral leiomyoma was made.
Routine blood investigations done were found to be normal. Trans Vaginal Ultra Sonogram suggested a rounded heterogeneous pelvic mass. Enucleation of the leiomyoma under regional anesthesia was planned and carried out with ease (Figure 1).

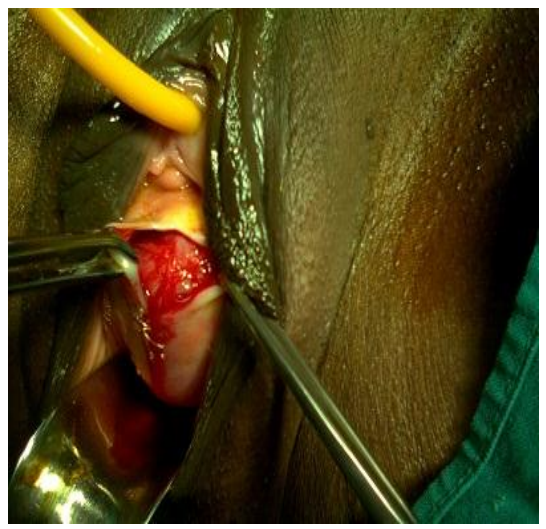

Figure 1: Intraoperative picture of the paraurethral leiomyoma.

Bladder was catheterized prior to enucleation for early identification of urethral injury. Patient withstood the procedure well and was discharged on fourth postoperative day. On follow-up, the patient was totally symptom free. Histopathological examination showed a well-encapsulated lesion, consisting of interlacing fascicles and bundles of spindle shaped smooth muscle 
cells, with elongated vesicular nuclei and moderate amounts of eosinophilic cytoplasm suggestive of leiomyoma (Figure 2).

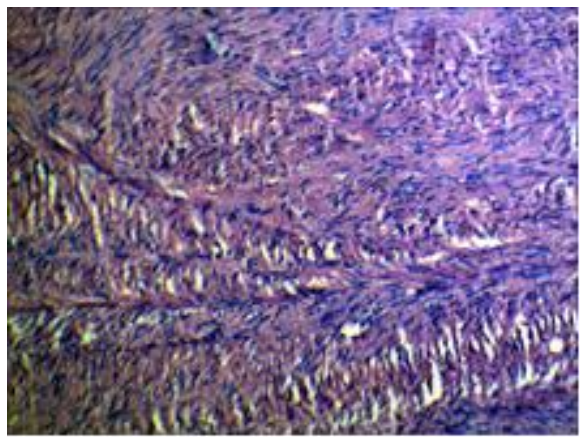

Figure 2: H \& E staining (10X)- slide showing interlacing fascicles and bundles of spindle shaped smooth muscle cells, with elongated vesicular nuclei and moderate amounts of eosinophilic cytoplasm, suggestive of leiomyoma.

\section{DISCUSSION}

Paraurethral tumours in the female are uncommon, so are leiomyomas. Embryologically, the urethra and vagina share a common origin. The female urethra is $3-4 \mathrm{~cm}$ long, extending from bladder to the vestibule traveling anterior to the vagina. It is lined by non-keratinizing squamous epithelium. Within the submucosa, lie the paraurethral glands. It also contains an inner longitudinal layer and outer circular layer of smooth muscle.

The vagina is a hollow fibromuscular tube, extending from the vulvar vestibule to the uterus. It is composed of 3 layers. Mucosal layer is made of non-keratinizing squamous epithelium without glands, muscularis made of connective tissue and smooth muscle, a loosely arranged inner circular and outer longitudinal layer of adventitia, consisting of endopelvic fascia, adherent to the underlying muscle.

Paraurethral leiomyomas usually present in the fourthfifth decade of life. Majority are asymptomatic (50\%), usually presenting as a painless mass, at the external genitalia. Women complain of local discomfort or a mass descending per vaginum. Urinary complaints like dysuria, incomplete voiding and urinary retention may be there. ${ }^{1}$ Dyspareunia is often encountered. One case with profuse bleeding from the leiomyoma has also been reported. ${ }^{2}$
Physical examination often reveals a firm, smooth nontender, non-fluctuant mass, which can be easily felt through the anterior vagina, as in our case. Paraurethral leiomyoma commonly arises from the posterior upper urethra. Size varies from few millimeters to few centimeters. However, a giant leiomyoma has been reported in the literature. ${ }^{3}$ The distinction between urethral, paraurethral and anterior vaginal wall leiomyoma may be difficult because of their close anatomic proximity.

Radiographic imaging studies are useful adjunct in the clinical evaluation of paraurethral leiomyoma. They are helpful to rule out any communication between the mass and the urinary tract. MRI can clinch the diagnosis, with a reasonable degree of certainty. ${ }^{4}$ However, if the mass is freely mobile, advanced radiographic techniques may be avoided.

Thorough review of literature suggests a surgical excision of these lesions. Approach through the anterior vaginal wall is safe without disturbing the urethra. Medical management is advocated, similar to uterine leiomyoma, in surgically unfit patients. No recurrences have been reported.

\section{Funding: No funding sources}

Competing interests: There are no competing interests to declare

Ethical approval: Not required

\section{REFERENCES}

1. Bruschini H, Truzzi JC, Simonetti R, et al. Paraurethral leiomyoma in a female causing urinary obstruction. Int Urogynecol J Pelvic Floor Dysfunct 2006;17:286-9.

2. Tantbirojn P, Sansopha L, Thankit V, Sirisubya N. Paraurethral leiomyoma: a case report. J Med Assoc Thai 2006;89:545-9.

3. Kuramoto $\mathrm{T}$, Nishizawa S, Mori $\mathrm{T}$, Iba A, et al. Giant paraurethral leiomyoma in the female: a case report. Hinyokika Kiyo 2008;54:677-80.

4. Hubert KC, Remer EM, Rackley RR, Goldman HB. Clinical and MRI characteristics of vaginal and paraurethral leiomyoma: can they be diagnosed before surgery? BJU Int 2010;105:1686-8.

DOI: $10.5455 / 2320-1770 . i j \operatorname{rog} 20130219$

Cite this article as: Sendhil CA, Ghose S, Samal R. Paraurethral leiomyoma in a woman: a case report. Int J Reprod Contracept Obstet Gynecol 2013;2:99-100. 